\title{
Patient-Specific Simulation of Pneumoperitoneum for Laparoscopic Surgical Planning
}

\author{
Shivali Dawda ${ }^{1}$ (I) $\cdot$ Mafalda Camara ${ }^{1} \cdot$ Philip Pratt $^{1} \cdot$ Justin Vale ${ }^{1} \cdot$ Ara Darzi $^{1} \cdot$ Erik Mayer $^{1}$
}

Received: 18 September 2018 / Accepted: 28 August 2019 / Published online: 10 September 2019

(C) The Author(s) 2019

\begin{abstract}
Gas insufflation in laparoscopy deforms the abdomen and stretches the overlying skin. This limits the use of surgical imageguidance technologies and challenges the appropriate placement of trocars, which influences the operative ease and potential quality of laparoscopic surgery. This work describes the development of a platform that simulates pneumoperitoneum in a patient-specific manner, using preoperative $\mathrm{CT}$ scans as input data. This aims to provide a more realistic representation of the intraoperative scenario and guide trocar positioning to optimize the ergonomics of laparoscopic instrumentation. The simulation was developed by generating 3D reconstructions of insufflated and deflated porcine CT scans and simulating an artificial pneumoperitoneum on the deflated model. Simulation parameters were optimized by minimizing the discrepancy between the simulated pneumoperitoneum and the ground truth model extracted from insufflated porcine scans. Insufflation modeling in humans was investigated by correlating the simulation's output to real post-insufflation measurements obtained from patients in theatre. The simulation returned an average error of $7.26 \mathrm{~mm}$ and $10.5 \mathrm{~mm}$ in the most and least accurate datasets respectively. In context of the initial discrepancy without simulation $(23.8 \mathrm{~mm}$ and $19.6 \mathrm{~mm})$, the methods proposed here provide a significantly improved picture of the intraoperative scenario. The framework was also demonstrated capable of simulating pneumoperitoneum in humans. This study proposes a method for realistically simulating pneumoperitoneum to achieve optimal ergonomics during laparoscopy. Although further studies to validate the simulation in humans are needed, there is the opportunity to provide a more realistic, interactive simulation platform for future image-guided minimally invasive surgery.
\end{abstract}

Keywords Simulation $\cdot$ Pneumoperitoneum $\cdot$ Patient-specific $\cdot$ Laparoscopy $\cdot$ Surgical planning

\section{Introduction}

Image guidance systems in surgery offer great potential to increase surgical accuracy and safety by augmenting the visualization of anatomical landmarks and subsurface structures during minimally invasive procedures. The utility of such technologies is often limited in laparoscopy due to the creation of pneumoperitoneum, which shifts the skin and deforms the

This article is part of the Topical Collection on Image \& Signal Processing

Shivali Dawda

shivali.dawda@gmail.com

1 Department of Surgery and Cancer, Imperial College London, London, UK abdominal wall, organs and blood vessels [1-3]. This also makes it challenging to ensure the optimal positioning of trocars, which is an essential determinant of the operative quality, safety and ease of laparoscopy that is presently based on the surgeon's experience and judgment of the post-insufflation operative field. Improper placement can result in poor laparoscopic view or instrumentation and poses an increased risk of vascular or organ damage. Modeling the changes that occur in the abdomen with gas insufflation is one way to overcome these issues, as well as facilitate surgical planning by providing a realistic, three-dimensional representation of the intraoperative scenario. It also offers the opportunity to enhance surgical training simulators and allow for guidance of trocar positioning in a way that optimizes the ergonomics of laparoscopic instrumentation.

Only a handful of groups have proposed methods for simulating pneumoperitoneum, from which a sufficient or desired technology has yet to surface. Previous groups have used traditional, physically-based methods of modeling dynamic 
objects which use internal and external forces to determine the positions of the displaced objects by time-integrating accelerations [4-8]. These techniques are typically highly complex and involve long computational times. In this work, biomechanical deformation is modeled with a position-based dynamics (PBD) approach, which simulates dynamic systems by calculating the displacement of objects to valid new positions such that constraints are satisfied [9]. As PBD works directly on the positions of objects (rather than with forces), it offers unconditional stability and can compute manipulations at interactive speeds (i.e. in real-time) with high visual fidelity that is especially suitable for complex surgical simulations [9]. Furthermore, using PBD entails a faster, more efficient data preparation protocol that favors simulation on a patientto-patient basis, whereas other approaches are highly time consuming and therefore not as feasible nor efficient for patient-specific planning. As the profile and extent of deformation to the abdominal wall and organs vary depending on each individual's physique, patient-specific modeling is highly advantageous.

This work is aimed at developing a platform that simulates the anatomical changes resulting from gas insufflation during laparoscopy in a patient-specific manner, using preoperative CT scans as the input data. This can assist surgeons in the planning and rehearsal of laparoscopic procedures by allowing realistic visualization and interaction with a virtual, 3-dimensional (3D) model of a specific patient's anatomy, post-insufflation. It will further serve to guide trocar positioning in a way that optimizes the ergonomics of laparoscopic instrumentation. This would allow for greater accuracy and utility of preoperative planning, which should ultimately improve surgical performance, decrease operation times and reduce error [10]. The simulation will be developed using a PBD approach on a porcine model, for practicability of obtaining insufflated and deflated volumetric data. Its feasibility for modeling insufflation in humans will be subsequently assessed by correlating the virtual simulated pneumoperitoneum to real post-insufflation measurements obtained from patients in theatre.

\section{Materials and methods}

3D models were generated from two sets of porcine data: insufflated scans and deflated scans. Models derived from the insufflated CT scans were considered ground truth. An artificial pneumoperitoneum was simulated on models from the deflated scans. The simulation parameters were optimized by comparing its output against the real pneumoperitoneum (derived from the insufflated porcine scans), and minimizing the difference.

\section{Data preparation: 3D reconstruction and mesh generation}

The datasets used were originally collected for other purposes in accordance with institutional guidelines, under the appropriate licenses, permissions and ethical approval. Eight pigs underwent gas insufflation of up to $12 \mathrm{mmHg}$ of abdominal
Fig. 1 Slices from insufflated and deflated porcine CT scans demonstrating the segmentation of different regions: gas (red), abdominal viscera (green), lungs (light blue) and abdominal wall (dark blue). The red line in the deflated scans indicates the boundary between the peritoneal cavity and the abdominal wall a) axial

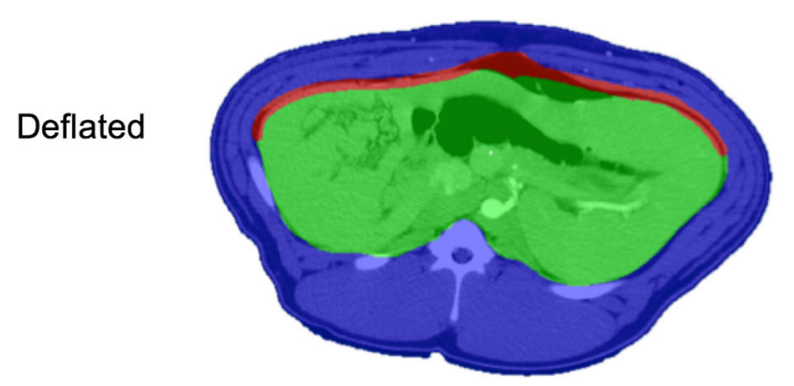

Insufflated

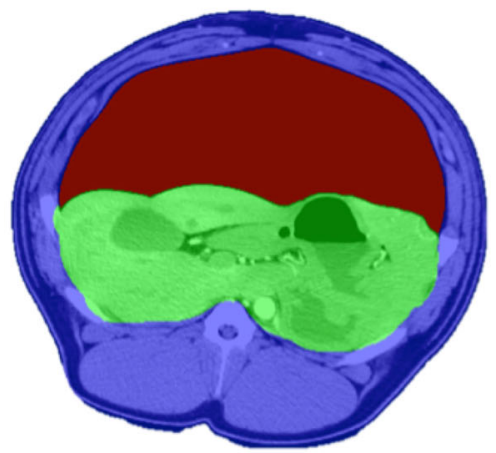

b) sagittal
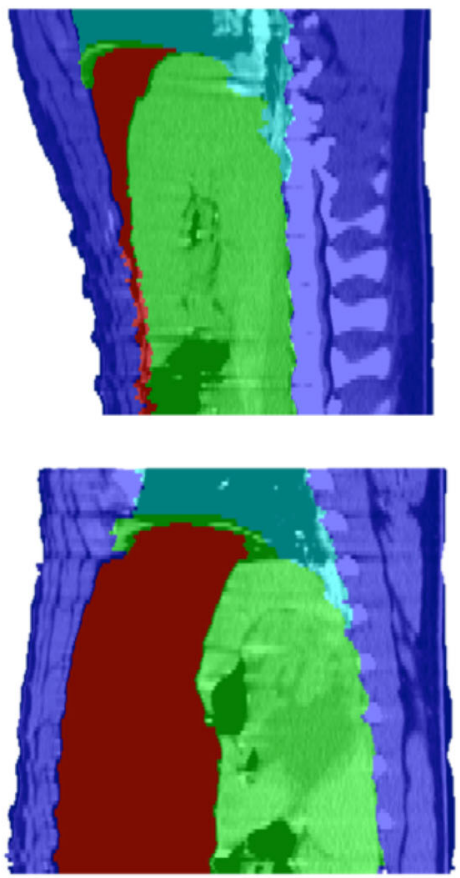
Fig. 2 3D reconstructions of an insufflated and deflated porcine scan, produced by interpolating individually segmented axial slices in Fig. 1a

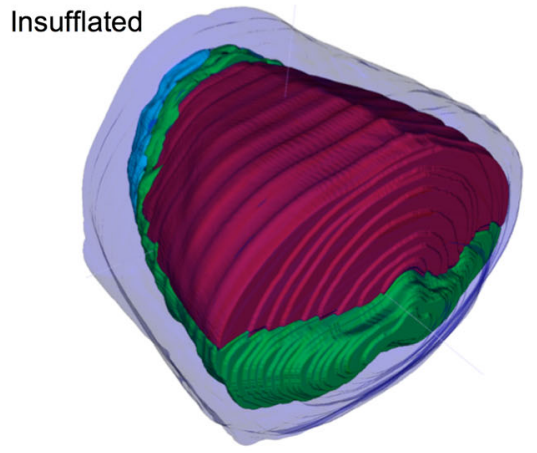

pressure. Acquisition of contrast-enhanced CT images ( $2.5 \mathrm{~mm}$ slice thickness, $512 \times 512$ acquisition matrix) was carried out with the animals in supine position, and repeated after deflation to produce two datasets for each pig: a deflated and insufflated volume.

3D reconstruction of preoperative scans can be produced through the process of manual or semi-automatic 'segmentation', whereby particular regions on a series of medical images are highlighted in different colors and interpolated in three dimensions to create a virtual model of a specific patient's anatomy (Figs. 1 and 2). Computed tomography (CT) provides sufficient information for abdominal reconstruction as the high spatial resolution prevents underlying tissues and structures from being superimposed [11]. 3D volume data was generated by segmenting axial slices of the original porcine CT scans in ITK-SNAP v3.6.0 [12] and extrapolating the model into a closed structure. CT images were divided into four regions (Fig. 1): the abdominal-thoracic wall (dark blue), abdominal viscera (green), pneumoperitoneum (red) and lungs (light blue). The rationale for segmenting organs collectively was based on previous attempts at simulating

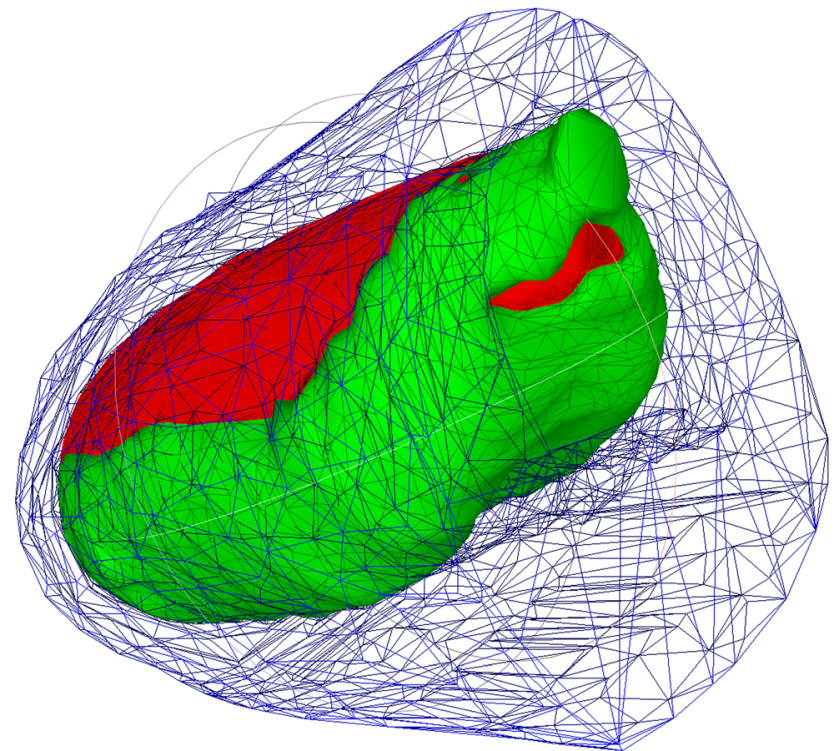

Fig. 3 Triangular surface meshes of insufflated porcine (abdominal wall in blue, organs in green, pneumoperitoneum in red) pneumoperitoneum, which produced acceptable results from modeling the abdominal viscera as a single homogenous structure [4-6].

3D segmentations were exported from ITK-SNAP into MeshLab (v2016.12) as triangular STL surface meshes (Fig. 3), where they were simplified (to around 10,000 triangular faces) and scaled down to half their size in order to speed up the calibration by inputting fewer particles for simulation, whilst preserving particle size [13].

\section{Simulation}

The abdominal wall and viscera were considered soft bodies, and the boundary between them regarded as an 'inflatable structure'. Each of these entities were entirely discretised into particles (Fig. 4) and modeled as separate structures by applying different simulation parameters to the particles. The inflatable structure was derived from highlighting the boundary between the abdominal wall and viscera in the segmentation step. This region represents the peritoneal cavity where gas is insufflated in laparoscopy, and is artificially inflated in the simulation by applying pressure from within the mesh (Fig. 5).

Gravity in the simulation was set to zero, and particles in a specific region of the back ( $3 \mathrm{~mm}$ below the axis defined by the center of mass) were fixed along the cranio-caudal axis in order to account for contact with the operating table (green points in Fig. 4). An exhaustive search was conducted to

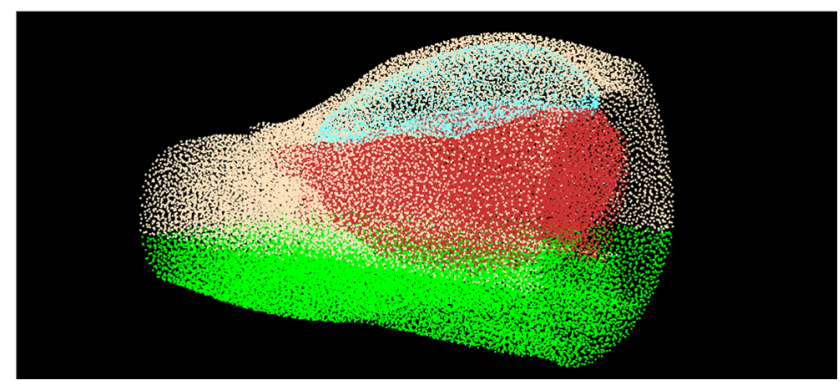

Fig. 4 Particle density of insufflated mesh, separated into the inflatable structure (blue), skin (peach), viscera (red). Green points indicate fixed regions in the back 
Fig. 5 Pneumoperitoneum before and after simulation, showing increased volume of the pneumoperitoneum $(10 \mathrm{x})$ and resultant organ compression and abdominal wall deformation
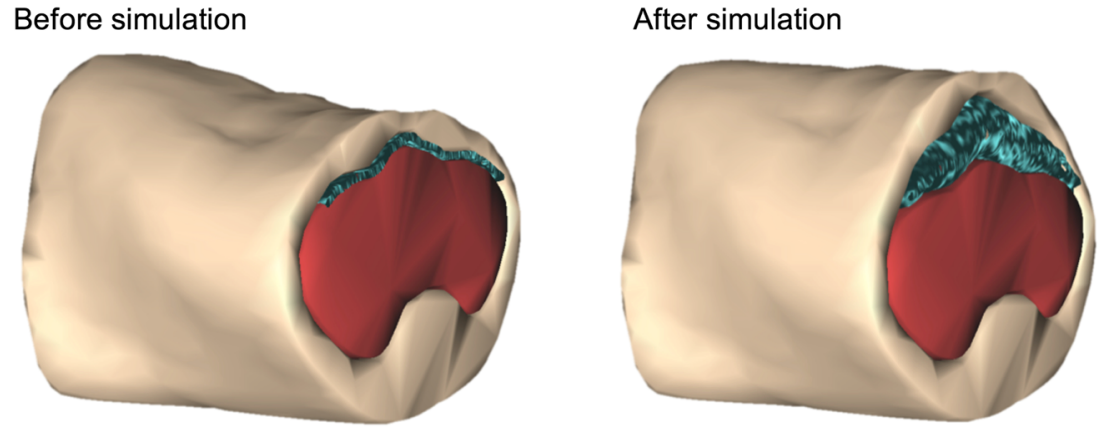

determine the optimal combination of parameters that would minimize the error in the simulated inflatable structure, when compared to the ground truth model derived from the insufflated porcine scan. Ranges adopted for each parameter were selected based on previous work involving soft tissue calibration [14] and experience of the simulation's sensitivity to certain parameters. Table 1 shows the simulation settings used during this calibration process.

\section{Optimization using porcine data}

Table 2 lists the parameters undergoing optimization. The pressure applied on the inflatable is increased and the simulation is performed for each value of pressure (from 1.0 to 10.0), which represents a proportionate increase in the original volume of the inflatable. This can in future be translated to a value of pressure in $\mathrm{mmHg}$ via a second calibration process whereby scans acquired over a range of insufflation pressures would provide a ground truth model for validation at each pressure.

The simulation was optimized by adopting the set of parameters that returned the minimal error when comparing simulated meshes with those derived from the insufflated porcine scans (ground truth). This comparison was made by calculating the mean Euclidean distances between corresponding

Table 1 Simulation settings for calibration of parameters

\begin{tabular}{ll}
\hline Time step & $1 / 60 \mathrm{~s}$ \\
Simulation substeps & 3 (collision detections per timestep) \\
Substep iterations & 9 \\
Cluster spacing factor & 3.33 (controls cluster overlapping) \\
Volume sampling factor & 4 (controls particle density) \\
Relaxation type & Local (relaxation factor $=1.0)$ \\
Acceleration due to gravity & $0 \mathrm{~m} / \mathrm{s}^{2}$ \\
Tissue density & $1.05 \mathrm{~g} / \mathrm{cm}^{3}$ \\
Shape friction coefficient & 0.35 \\
Particle friction coefficient & 0.25 \\
Damping factor & 12.0 \\
\hline
\end{tabular}

points on the simulated meshes and the ground truth meshes across all vertices, for the entire porcine dataset. It is this quantity that underwent minimization during optimization. The resultant set of parameters are summarized in Table 3.

To avoid undesirable behavior, such as the pneumoperitoneum expanding outside the abdominal wall, extra springs were added into the simulation to assure connectivity between the inflatable structure and the abdominal wall without invalidating the resulting set of optimized parameters. The optimized simulation was performed on each animal for the given value of pressure with which they were inflated during CT acquisition. Meshes representing the abdominal wall, the viscera and the pneumoperitoneum were extracted, as well as the mean error, standard deviation and minimum and maximum errors (Euclidian distances).

\section{Feasibility of modeling pneumoperitoneum in humans}

As well as giving unnecessary exposure to radiation, it is impractical to scan patients whilst maintaining pneumoperitoneum for direct comparison to a simulation. Insufflation modeling in humans was therefore assessed by correlating the simulation's output to real post-insufflation measurements obtained from patients in undergoing laparoscopic surgery (Fig. 6). Landmarks were chosen for their accessibility through sterile drapes and visibility on CT images. Under the existing ethical protocol 'Improving Outcomes in Robotic and Endoscopic Surgery using Augmented Reality Guidance' (REC reference 07/Q0703/24), informed and written consent was obtained from patients recruited to the study.

\section{Results}

\section{Validation on porcine data}

Using the resultant optimal set of parameters, pneumoperitoneum was simulated for each pig by increasing the volume of the inflatable structure. Volume is proportional to the 
Table 2 Parameters for optimization

\begin{tabular}{|c|c|c|}
\hline Parameter & Influence & Range \\
\hline Cluster stiffness & $\begin{array}{l}\text { Controls stiffness and deformability } \\
\text { of soft tissues }\end{array}$ & $0.4,0.5,0.6,0.7,0.8$ \\
\hline Spring stiffness & $\begin{array}{l}\text { Controls stiffness of the inflatable structure } \\
\text { and its resultant deformability }\end{array}$ & $0.1,0.2,0.3,0.4,0.5,0.6,0.7,0.8,0.9,1.0$ \\
\hline Particle radius & $\begin{array}{l}\text { Determines the size of each particle, directly } \\
\text { influencing the number of particles that } \\
\text { comprise an object }\end{array}$ & $2.2 \mathrm{~mm}, 2.7 \mathrm{~mm}, 3.3 \mathrm{~mm}$ \\
\hline Simulation pressure & $\begin{array}{l}\text { Proportionate to the increase in the original } \\
\text { volume of the inflatable }\end{array}$ & $\begin{array}{l}1.0,1.5,2.0,2.5,3.0,3.5,4.0,4.5,5.0,5.5,6.0,6.5,7.0,7.5,8.0,8.5 \\
\quad 9.0,9.5,10.0,10.5,11.0,11.5,12.0,12.5,13.0,13.5,14.0,14.5,15.0\end{array}$ \\
\hline
\end{tabular}

simulation pressure and hence labeled "simulation pressure factor". The overall mean error in the simulated meshes was determined by calculating the Euclidean distance between corresponding points on the simulated pneumoperitoneum and ground truth models. This was plotted for each pressure value, for each pig (Fig. 7). The simulation produced the best results in the 7 th porcine dataset, which gave the lowest overall error (7.26 mm). Conversely, the 2 nd dataset was the least successful, returning the highest overall error $(10.5 \mathrm{~mm})$. The initial displacement, calculated before any simulation pressure was applied, was $23.8 \mathrm{~mm}$ and $19.6 \mathrm{~mm}$ for the most and least accurate simulations respectively.

All datasets followed a general trend whereby the mean overall error decreased until it reached a minimum, at which point the simulated pneumoperitoneum was most aligned with the ground truth meshes. Increasing the pressure beyond this minimum began to increase the overall error, showing that the simulation was over-expanding the inflatable structure. Curves displayed variable behavior in reaching their minimum error at different simulation pressure values. Table 4 gives a summary of the most and least accurate simulations.

Errors were derived using an absolute distance function and are illustrated in Fig. 8 on color maps of the simulated inflatable structures. The most and least successful simulations are shown for contrast; Fig. 8a illustrates the average error in the well-simulated 7 th dataset $(7.26 \mathrm{~mm})$ whereas Fig. $8 \mathrm{~b}$ demonstrates the same concept in the least accurate 2nd dataset $(10.5 \mathrm{~mm})$. Error in the 2 nd dataset is evident in the red region, where the inflatable structure has expanded outside of the wireframe of the abdominal wall.

Table 3 Optimized parameters

\begin{tabular}{ll}
\hline Parameter & Optimal value \\
\hline Cluster stiffness & 0.6 \\
Spring stiffness & 0.5 \\
Particle radius & $2.7 \mathrm{~mm}$ \\
Simulation pressure & 8.9 \\
\hline
\end{tabular}

\section{Human simulation}

Human CT scans were successfully segmented and simulated for pneumoperitoneum. Pre- and post-insufflation measurements were collected from theatre and from the generated anatomic models (Table 5).

\section{Discussion}

Segmentations of the porcine dataset were sufficient to derive an optimal set of parameters for the simulation. The simulation was successful in realistically modeling organ and abdominal wall deformation, with an average error of $7.26 \mathrm{~mm}$ in the most accurate simulation. This "error" refers to the

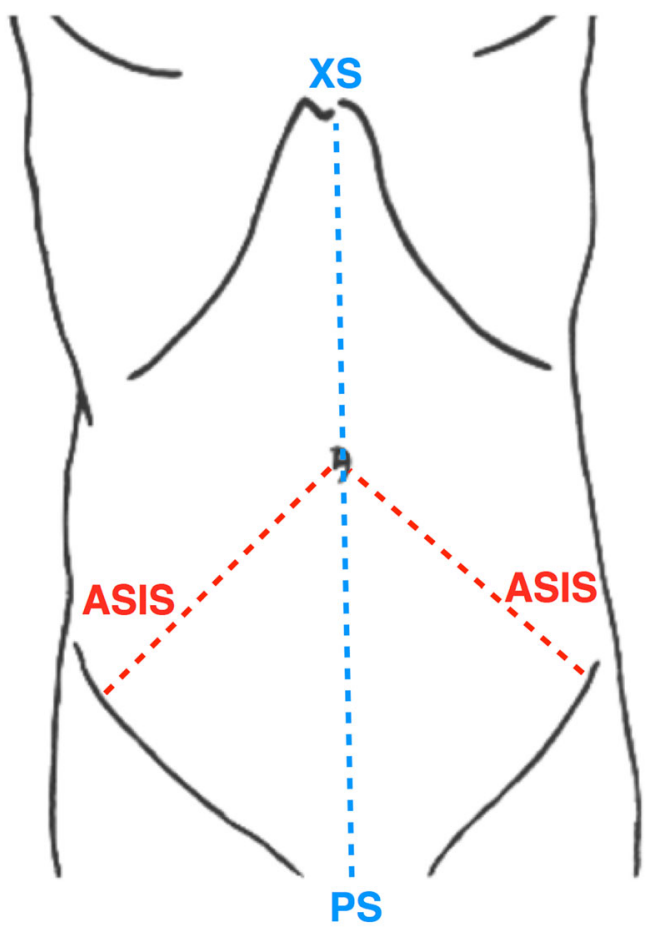

Fig. 6 Three measurements were taken from landmarks on the abdominal surface: umbilicus to right and left anterior-superior iliac spines (ASIS), xiphisternum (XS) to pubic symphysis (PS) 


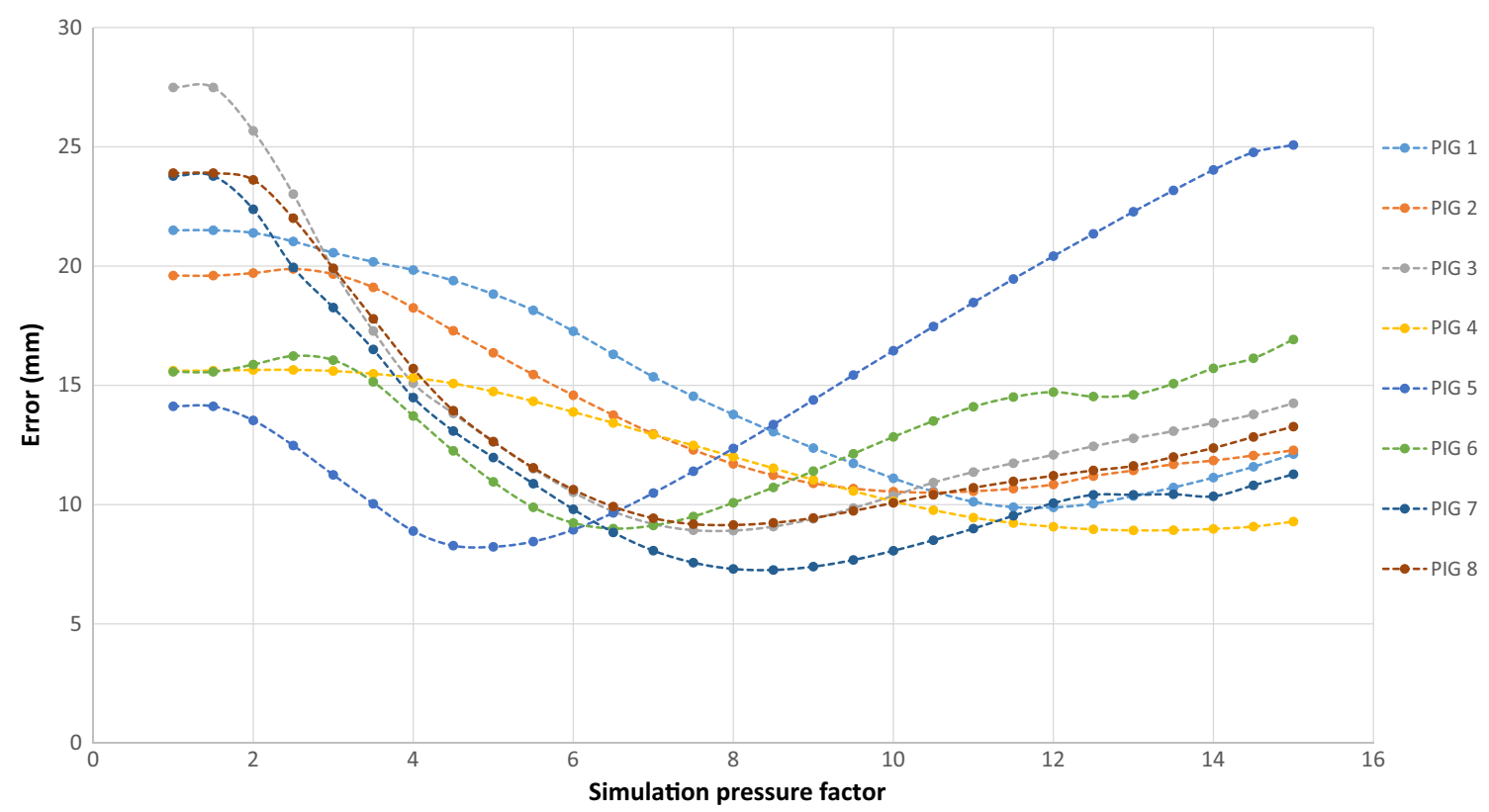

Fig. 7 Mean overall error of simulated meshes across simulation pressure, as average distance to corresponding vertices on ground truth meshes from insufflated porcine scans

overall difference between the simulation's output, when compared against the real-life inflated porcine. This must be interpreted in context of the original discrepancy between the insufflated and deflated porcine, which was calculated to be $23.8 \mathrm{~mm}$. This initial displacement, present before any pressure was simulated, represents the "error" that surgeons currently need to operate with. The threefold reduction in error shows that the methods proposed here have provided a significantly improved picture of the intraoperative scenario.

The porcine model has good translatability for human simulation. Pigs are the preferred animate trainers for complex laparoscopic techniques as the size of their abdominal cavity and their foregut anatomy is similar to that of humans, which provides comparable ergonomics to human laparoscopy and allows for the creation of pneumoperitoneum [15]. The muscle layers that formed the boundaries of the abdominal cavity in this simulation are organized in a similar fashion in both pigs and humans [16].
A major issue in the field of patient-specific biomechanical modeling is how to reproduce clinically accurate simulations without knowledge of the patient-specific mechanical properties of tissues. Abdominal deformation by pneumoperitoneum varies by age, sex, BMI and other patient variables. However, Miller et al. demonstrate that it is possible to achieve, for the purpose of this application, a realistic prediction of tissue deformations using preoperative images alone [17]. A patientspecific anatomic response to increasing abdominal pressure can therefore be calculated using solely the geometry of the abdominal wall - which is obtained from the segmentation of preoperative CT image data as described. This suggests the effect of patient mechanics on abdominal deformation by pneumoperitoneum can be disregarded.

When compared to previous works, this simulation models pneumoperitoneum with respectable accuracy. Oktay et al. achieved an initial average error of $10.9 \mathrm{~mm}$ (before image registration) when validated on 3 porcine CT-scans [7]. Bano et al. simulated movement of the abdominal wall and viscera
Table 4 Average error of most and least accurate simulations

\begin{tabular}{lll}
\hline Simulation & Most accurate (pig 7) & Least accurate (pig 2) \\
\hline Average overall error (mm) & 7.26 & 10.5 \\
Standard deviation (mm) & 2.15 & 2.77 \\
Minimum (mm) & 0.158 & 0.190 \\
Maximum (mm) & 15.2 & 16.2 \\
Initial displacement - before simulation (mm) & 23.8 & 19.6 \\
\hline
\end{tabular}


Fig. 8 Color maps displaying map illustrating the overall error $(\mathrm{mm})$ of the simulated pneumoperitoneum of the 2 nd and 7 th porcine datasets at their optimal pressure parameters. Colors correspond to the overall error in millimeters (as on the color bar). Warmer colors indicate a higher degree of misalignment with the ground truth mesh, implying greater overall error a) most accurate simulation

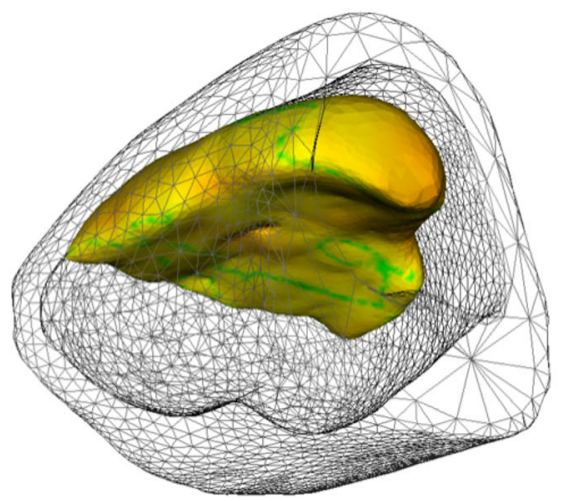

b) least accurate simulation

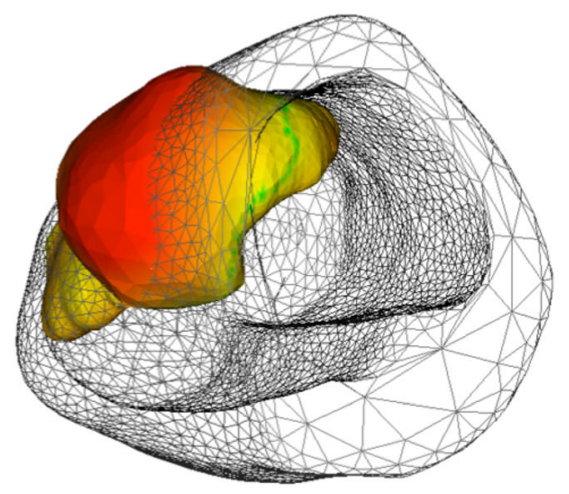

$\operatorname{Error}(\mathrm{mm})$

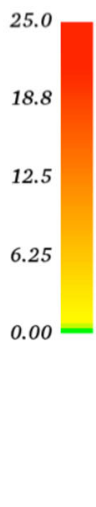

with $5 \mathrm{~mm}$ and $6 \mathrm{~mm}$ accuracy respectively from validation in 2 pigs [5], and Nimura et al. report an average error of $26.9 \mathrm{~mm}$ from comparing their models to the displacement of optically-sensed points on human abdominal surface [8]. The minimum and maximum average errors obtained from this simulation was $7.26 \mathrm{~mm}$ and $10.5 \mathrm{~mm}$ respectively. These results were obtained from a much larger dataset (eight pigs) than any previous work. It provides the added speed and unconditional stability of PBD, which gives the simulation promising applications due to its high visual fidelity and ability to compute deformations in real-time. Furthermore, a realistic, patient-specific simulation of human pneumoperitoneum has been demonstrated using a technology that works at interactive speeds that is feasible for large-scale use.

This study has some weaknesses. Certain sources of error may have contributed to simulation inaccuracy: the study protocol involved a large amount of data processing, which is liable to the loss of precision despite being handled mostly by the same individual. To preserve the simulation's generalizability, pigs were not normalized for size during calibration, which may reflect in the data: results suggest a possible relationship between elements of the pig's geometrical features and the optimal simulation parameters. Furthermore, a robust validation for human simulation is required. This could be achieved by acquiring more accurate measurements of pneumoperitoneum, such as intraoperative imaging or optical position sensing of markers placed on the abdominal surface $[3$,
8]. Regardless, this work has demonstrated that human preoperative medical images can be successfully processed for real time, 3D, patient-specific simulation.

The simulation's performance can be improved through various approaches. Future studies could acquire CT scans across a range of different insufflation pressures to ensure there is accurate modeling of the rate of organ deformation. Furthermore, as the simulation was developed and tested on the same dataset of eight pigs, it should be validated on a new dataset. Incorporating a gravity-compensation study would enable the framework to simulate pneumoperitoneum even when the patient is lying on their side, despite CT acquisition of them lying supine. This would increase its application to a variety of positions - e.g. in urology, where patients are positioned on their side for laparoscopic nephrectomies. Also, modeling the organs individually could produce greater accuracy that would be beneficial for more detailed surgical image guidance (e.g. for liver resections).

Next steps would involve using the simulation to inform and enhance surgical planning. The simulation could be integrated into virtual reality simulators used in the training of laparoscopic surgeons to create a highly realistic, patient-specific training environment for operation rehearsal [18]. The stability and speed of PDB allows surgeons to interact with realistically insufflated, virtual models of patient anatomy in real time, giving them the opportunity to define and rehearse their surgical strategy on a case-by-case basis depending on the patient and target
Table 5 Abdominal surface changes from insufflation: human data

\begin{tabular}{|c|c|c|c|c|c|c|}
\hline & \multicolumn{3}{|c|}{ Intraoperative data $(\mathrm{cm})$} & \multicolumn{3}{|c|}{ Simulation $(\mathrm{cm})$} \\
\hline & $\begin{array}{l}\text { Umbilicus } \\
\text { to ASIS-R }\end{array}$ & $\begin{array}{l}\text { Umbilicus } \\
\text { to ASIS-L }\end{array}$ & $\begin{array}{l}\text { Xiphisternum to } \\
\text { pubic symphisis }\end{array}$ & $\begin{array}{l}\text { Umbilicus } \\
\text { to ASIS-R }\end{array}$ & $\begin{array}{l}\text { Umbilicus } \\
\text { to ASIS-L }\end{array}$ & $\begin{array}{l}\text { Xiphisternum to } \\
\text { pubic symphisis }\end{array}$ \\
\hline Normal & 18 & 17.5 & 32.8 & 17.5 & 18.4 & 39.3 \\
\hline Insufflated & 19 & 17.5 & 37.4 & 18.9 & 20.1 & 41.9 \\
\hline Change & 1 & 0 & 4.6 & 1.4 & 2.3 & 2.6 \\
\hline
\end{tabular}


organ. Augmented reality (AR), which involves the addition of virtual elements to a real scene, has recently become a popular area of development in the laparoscopic community and in image-guided surgery. However, the utility of AR in laparoscopy is largely limited: insufflating the abdomen complicates image registration and makes intraoperative anatomy inconsistent to 3D reconstructions of preoperative scans [19]. Modeling insufflation offers the opportunity to overcome these discrepancies, which have been a major obstacle in the use of AR as an image guidance tool in laparoscopy so far [20].

Altogether, this highlights how image guidance systems in laparoscopy could hugely benefit from patient-specific simulation of pneumoperitoneum. This research presents a method for realistically simulating pneumoperitoneum using preoperative images as the input data. It aims to facilitate surgical planning, as well as provide a more realistic platform for future image guidance in laparoscopy.

Acknowledgements This work is independent research funded by the National Institute for Health Research (NIHR) Imperial Biomedical Research Centre (BRC). The views expressed in this publication are those of the author(s) and not necessarily those of the NHS, the National Institute for Health Research or the Department of Health. Thanks to Dr. Ismail Omar, Dr. James Dilley, Dr. Alasdair Scott and Mr. James Kinross.

Funding Information This work is independent research funded by the National Institute for Health Research (NIHR) Imperial Biomedical Research Centre (BRC). The views expressed in this publication are those of the author(s) and not necessarily those of the NHS, the National Institute for Health Research or the Department of Health.

\section{Compliance with ethical standards}

Conflict of interest The authors declare that they have no conflict of interest.

Ethical approval All procedures performed in studies involving human participants were in accordance with the ethical standards of the institutional and/or national research committee and with the 1964 Helsinki declaration and its later amendments or comparable ethical standards. All applicable international, national, and/or institutional guidelines for the care and use of animals were followed.

Open Access This article is distributed under the terms of the Creative Commons Attribution 4.0 International License (http:// creativecommons.org/licenses/by/4.0/), which permits unrestricted use, distribution, and reproduction in any medium, provided you give appropriate credit to the original author(s) and the source, provide a link to the Creative Commons license, and indicate if changes were made.

\section{References}

1. Sánchez-Margallo, F. M., Moyano-Cuevas, J. L., Latorre, R., Maestre, J., Correa, L., Pagador, J. B. et al., Anatomical changes due to pneumoperitoneum analyzed by MRI: An experimental study in pigs. Surg. Radiol. Anat. 33:389-396, 2011. https://doi. org/10.1007/s00276-010-0763-9.

2. Elliott, R. C., and Kirberger, R. M., Computed tomography determined changes in position of the urogenital system after $\mathrm{CO}_{2}$ insufflation to determine optimal positioning for abdominal laparoscopy. Vet. Surg. 44:91-99, 2015. https://doi.org/10.1111/vsu.12351.

3. Song, C., Alijani, A., Frank, T., Hanna, G. B., and Cuschieri, A., Mechanical properties of the human abdominal wall measured in vivo during insufflation for laparoscopic surgery. Surg. Endosc. 20:987-990, 2006. https://doi.org/10.1007/s00464-005-0676-6.

4. Kitasaka, T., Mori, K., Hayashi, Y., Suenaga, Y., Hashizume, M., and Toriwaki, J., Virtual pneumoperitoneum for generating virtual laparoscopic views based on volumetric deformation. In: Barillot $C$, Haynor DR, Hellier P, editors. Med. Image Comput. Comput. Interv. - MICCAI 2004 7th Int. Conf. Saint-Malo, Fr. Sept. 2629, 2004. Proceedings, part II, Berlin: Springer Berlin Heidelberg; 2004, 559-567. https://doi.org/10.1007/978-3-540-30136-3_69.

5. Bano, J., Hostettler, A., Nicolau, S. A., Cotin, S., Doignon, $\bar{C}$., Wu, H. S. et al., Simulation of pneumoperitoneum for laparoscopic surgery planning. Med. Image Comput. Comput. Interv. - MICCAI 2012, 2012, 91-98.

6. Bano, J., Hostettler, A., Nicolau, S. A., Doignon, C., Wu, H. S., Huang, M. H., et al., Simulation of the abdominal wall and its arteries after pneumoperitoneum for guidance of port positioning in laparoscopic surgery. In: Bebis G, Boyle R, Parvin B, Koracin D, Fowlkes C, Wang S, et al., editors. Adv. Vis. Comput. 8th Int. Symp. ISVC 2012, Rethymnon, Crete, Greece, July 16-18, 2012, Revis. Sel. Pap. Part I, Berlin: Springer Berlin Heidelberg; 2012, 111. https://doi.org/10.1007/978-3-642-33179-4_1.

7. Oktay, O., Zhang, L., Mansi, T., Mountney, P., Mewes, P., Nicolau, S., et al., Biomechanically driven registration of pre- to intraoperative 3D images for laparoscopic surgery. Lect. Notes Comput. Sci. (including Subser. Lect. Notes Artif. Intell. Lect. Notes Bioinformatics), vol. 8150 LNCS, 2013, 1-9. https://doi. org/10.1007/978-3-642-40763-5_1.

8. Nimura, Y., Di Qu, J., Hayashi, Y., Oda, M., Kitasaka, T., Hashizume, M. et al., Pneumoperitoneum simulation based on mass-spring-damper models for laparoscopic surgical planning. J. Med. Imaging 2:44004, 2015. https://doi.org/10.1117/1.JMI.2. 4.044004.

9. Müller, M., Heidelberger, B., Hennix, M., and Ratcliff, J., Position based dynamics. J. Vis. Commun. Image Represent. 18:109-118, 2007. https://doi.org/10.1016/j.jvcir.2007.01.005.

10. Zevin, B., Aggarwal, R., and Grantcharov, T. P., Surgical simulation in 2013: Why is it still not the standard in surgical training? J. Am. Coll. Surg. 218:294-301, 2014. https://doi.org/10.1016/j. jamcollsurg.2013.09.016.

11. Goldman, L. W., Principles of CT: Radiation dose and image quality. J. Nucl. Med. Technol. 35:213-225-228, 2007. https://doi.org/ 10.2967/jnmt.106.037846.

12. Yushkevich, P. A., Piven, J., Hazlett, H. C., Smith, R. G., Ho, S., Gee, J. C. et al., User-guided 3D active contour segmentation of anatomical structures: Significantly improved efficiency and reliability. Neuroimage 31:1116-1128, 2006. https://doi.org/10.1016/ j.neuroimage.2006.01.015.

13. Cignoni, P., Cignoni, P., Callieri, M., Callieri, M., Corsini, M., Corsini, M., et al. MeshLab: An open-source mesh processing tool. Sixth eurographics ital chapter conf 2008:129-136. https://oi.org/ 10.2312/LocalChapterEvents/ItalChap/ItalianChapConf2008/129136.

14. Camara, M., Mayer, E., Darzi, A., and Pratt, P., Soft tissue deformation for surgical simulation: A position-based dynamics approach. Int. J. Comput. Assist. Radiol. Surg. 11:919-928, 2016. https://doi.org/10.1007/s11548-016-1373-8.

15. Sung, G. T. , and Sun, Y., Animal laboratory training: Current status and how essential is it? In: Hemal AK, Menon M, editors. Robot. 
Genitourin. Surg., London: Springer London; 2011, 147-156. https://doi.org/10.1007/978-1-84882-114-9 12.

16. Dondelinger, R. F., Ghysels, M. P., Brisbois, D., Donkers, E., Snaps, F. R., Saunders, J. et al., Relevant radiological anatomy of the pig as a training model in interventional radiology. Eur. Radiol. 8:1254-1273, 1998. https://doi.org/10.1007/s003300050545.

17. Miller, K., and Lu, J., On the prospect of patient-specific biomechanics without patient-specific properties of tissues. J. Mech. Behav. Biomed. Mater. 27:154-166, 2013. https://doi.org/10.1016/ j.jmbbm.2013.01.013.

18. Kim, H. L., and Schulam, P., The PAKY, HERMES, AESOP, ZEUS, and da Vinci robotic systems. Urol. Clin. N. Am. 31:659669, 2004. https://doi.org/10.1016/j.ucl.2004.06.008.

19. Sugimoto, M., Yasuda, H., Koda, K., Suzuki, M., Yamazaki, M., Tezuka, T. et al., Image overlay navigation by markerless surface registration in gastrointestinal, hepatobiliary and pancreatic surgery. J. Hepatobiliary Pancreat. Sci. 17:629-636, 2010. https://doi.org/ 10.1007/s00534-009-0199-y.

20. Bernhardt, S., Nicolau, S. A., Soler, L., and Doignon, C., The status of augmented reality in laparoscopic surgery as of 2016. Med. Image Anal. 37:66-90, 2017. https://doi.org/10.1016/j.media. 2017.01.007.

Publisher's Note Springer Nature remains neutral with regard to jurisdictional claims in published maps and institutional affiliations. 